\title{
An Innovative Approach to Improve Diabetes Management in Accountable Care Organizations
}

\author{
Chris E. Stout ${ }^{1 *}$ and Grace Wang ${ }^{2}$ \\ ${ }^{1}$ Department of Research and Data Analytics, ATI Physical Therapy, University of Illinois, Chicago, United States \\ ${ }^{2}$ CPM/Caseloads Administrator, ATI Physical Therapy, Remington Blvd. Bolingbrook, Illinois, United States
}

Received: April 11, 2014; Accepted: April 14, 2014; Published: April 15, 2014

*Corresponding author: Chris E. Stout, Department of Research and Data Analytics at ATI Physical Therapy, Clinical Professor, University of Illinois, College of Medicine, Chicago, USA Email: chris.stout@atipt.com

As the Affordable Care Act becomes more widely operational across the United States, the expectation is that all citizens will benefit from better access to quality healthcare. This broadbased prospect of benefit is especially presumed to improve the access to care for those with diabetes. One of the key offerings of this current reform is the development of accountable care organizations (ACOs), which focus more so on populations within a region of a hospital center. The additional expected patient benefits of ACOs include better access to care and coordination of that care.

Perhaps one of the ways this will occur is less obvious to many that is, the relationship of the physical therapist and diabetes care. In an ACO, a patient may first present to physical therapy for care for something that may or may not be related to the diabetic condition. In the intake process, the physical therapist would take a medical history and if a patient presented with diabetes and was not already under the care of a physician, he or she could make a referral for such within the ACO structure. One of the most critical aspects of increased morbidity and mortality is untreated diabetes. In a study conducted in our organization, it was discovered that somewhere between $7-16 \%$ of patients presenting for physical therapy services did not have a primary care physician (PCP) and anywhere from $7-26 \%$ of patients had not seen their PCP in the last twelve months. Furthermore, almost $10 \%$ of currently active patients have noted diabetes as a co-morbidity.

Indeed, a number of issues are sequelae of diabetes, including increased chance of recurrent adhesive capsulitis, heart disease, stroke, hypertension, blindness and other eye complications, kidney disease, nervous system disease, dental disease, and pregnancy complications [1,2]. Frykberg et al. [3] note "ulcerations, infections, Charcot neuroarthropathy, and peripheral arterial disease frequently result in gangrene and lower limb amputation. Consequently, foot disorders are leading causes of hospitalization for persons with diabetes and account for billion-dollar expenditures annually in the US. Although not all foot complications can be prevented, dramatic reductions in frequency have been achieved by taking a multidisciplinary approach to patient management." We could not agree more. It is only through an integrated multidisciplinary approach that optimal outcomes can be achieved, and hopefully through the new mechanisms offered by ACOs and the Affordable Care Act they will now be available to those in need, as well as better managing concomitant costs.

One last example of this integration is demonstrated in our procedure to screen all of our Medicare patients for their Body Mass Index. Those flagged as exceeding normal limits are provided with information to assist in gaining a nutritional consultation a potential preventative measure for those at risk of diabetes-onset. Integrating these aspects into routine physical therapy practice is not onerous and indeed demonstrates more of a comprehensive approach to wellness focused care. We believe such holistic, synthetic, and integrative approaches will also help to tear down the iatrogenic silos that often exist between differing disciplines or specialties in medicine and healthcare today. This, in tandem with improved patient identification and along with access and integrated provider payment, all bode well to truly impacting diabetes care perhaps more than any other time in our history.

\section{References}

1. Maricar N, Shacklady C., McLoughlin L (2009) Effect of Maitland mobilization and exercises for the treatment of shoulder adhesive capsulitis: a single-case design. Physiother Theory Pract. 25(3): 213217.

2. National diabetes fact sheet (2011) national estimates and general information on diabetes and prediabetes in the United States. Centers for Disease Control and Prevention Web site. Published 2011. Accessed April 9, 2014.

3. Frykberg RG, Zgonis T, Armstrong DG, Driver VR, Giurini JM et al. (2006) Diabetic foot disorders: A clinical practice guideline. J Foot Surg. 45(5): S1-S66.

Citation: Stout CE, Wang G (2014) An Innovative Approach to Improve Diabetes Management in Accountable Care Organizations. J Endocrinol Diab 1(1): 2. http://dx.doi.org/10.15226/2374-6890/1/1/00105 